\title{
HANDBOOK OF CRITICAL ISSUES IN FINANCE
}

Edited by Jan Toporowski and Jo Michell, University of London, UK

This vital new Handbook is an authoritative volume presenting key issues in finance that have been widely discussed in the financial markets but have been neglected in textbooks and the usual compilations of conventional academic wisdom.

A wide range of topics including the recent economic crisis, capital controls, the Franc Zone, quantitative easing and securitization, as well as the key controversies associated with them, are explored and explained in depth by well-known authorities in finance and economics.

Designed to complement and expand upon standard textbooks as well as the specialist critical literature on particular topics in finance, this informative Handbook will prove invaluable to academics, researchers and students focusing on economics, finance and heterodox economics.

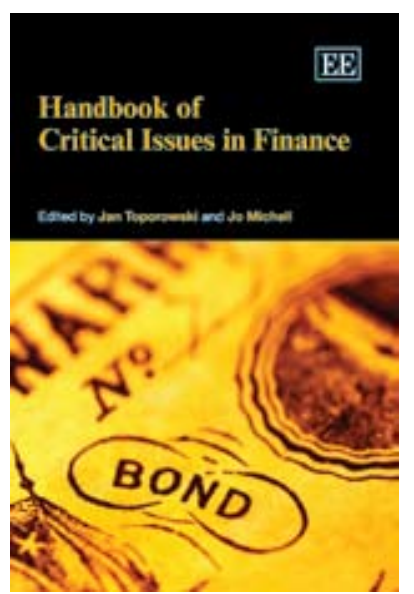

Contributors include: P. Arestis, R. Bellofiore, D. Bezemer, S. Blankenburg, H. Braun, T. Congdon, G. Cozzi, P. dos Santos, S. Dow, T. Evans, G. letto-Gillies, P. Kalmi, A. Kaltenbrunner, E. Karwowski, J. Kregel, S. Krishnan, M. Lawlor, C.G. Leathers, N. Levy Orlik, P. Lysandrou, D. Mayes, J. Michell, T. Mott, A. Nesvetailova, J.P. Painceira, R. Palan, J. Perraton, J. Powell, P. Raines, K. Ruziev, W. Song, E. Stockhammer, J. Toporowski, A. Trigg, E. Tymoigne, J. Tyson, L. Ventimiglia, S. Venugopalan, R. Wade, C. Whalen, M. Wolfson, G. Wood, L.R. Wray

\section{6 pp Hardback $9781849803700 £ 120.00$ • (e) 9781849805957}

ELGAR ORIGINAL REFERENCE • Full contents - www.e-elgar.com

\section{Contents:}

Introduction

1. Asian Monetary Union Wei Song

2. Bad Banks David G. Mayes

3. Bank Regulation Geoffrey Wood

4. Capital Controls Giovanni Cozzi

5. Capital Market Inflation Henryk Braun

6. Central Banks Tim Congdon

7. Central Bank Policy Juan Pablo Painceira

8. Commodity Markets Luigi Ventimiglia

9. Co-operative Banking Panu Kalmi

10. Credit Cycles Dirk Bezemer

11. Emerging Markets Kobil Ruziev

12. Microfinance Judith Tyson

13. The Exchange Rate Annina Kaltenbrunner

14. Financial Crises Martin H. Wolfon
15. Financial Fragility Eric Tymoigne

16. Financial Keynesianism Riccardo Bellofiore

17. Financial Markets In Developing Countries Noemi Levy Orlik

18. Financialization Engelbert Stockhammer

19. The Flow of Funds Jo Michell

20. Globalisation Jonathon Perraton

21. Hedge Funds Photis Lysandrou

22. The Iceland Crisis Robert H. Wade and Silla Sigurgeirsdottir

23. Inflation Targeting Philip Arestis

24. International Banking Trevor Evans

25. International Finance Jeff Powell

26. Islamic Banking Ewa Karwowski

27. John Maynard Keynes Michael S. Lawlor

28. Limited Liability Stephanie Blankenburg

29. Liquidity Anastasia Nesvetailova

30. Karl Marx
Andrew Trigg

31. The Methodology Of Finance Sheila C. Dow

32. Hyman P. Minsky Jan Kregel

33. Money In Finance L. Wrandall Wray

34. Money Manager Capitalism Charles J. Whalen

35. Option Pricing Models Paulo L. dos Santos

36. Overcapitalisation Jan Toporowski

37. Private Equity Funds Jan Toporowski

38. Quantitative Easing Shujoya Venugopalan

39. Risk Tracy Mott

40. Securitisation Sanjay Krishnan

41. Tax Havens Ronen Palan

42. Transnational Companies and Finance Grazia letto-Gillies

43. Thorstein Veblen Charles G. Leathers and J. Patrick Raines
TO ORDER, PLEASE CONTACT:

Marston Book Services Ltd 160 Milton Park

Abingdon, Oxon

OX14 4SD UK

Tel: + 441235465500

Fax: + 441235465555

direct.order@marston.co.uk

FOR INFORMATION, PLEASE CONTACT:

Sales \& Marketing Department Edward Elgar Publishing Ltd The Lypiatts

15 Lansdown Road

Cheltenham, Glos

GL50 2JA UK

Tel: + 441242226934

Fax: + 441242262111

www.e-elgar.com

for your free catalogues, please email:

info@e-elgar.co.uk

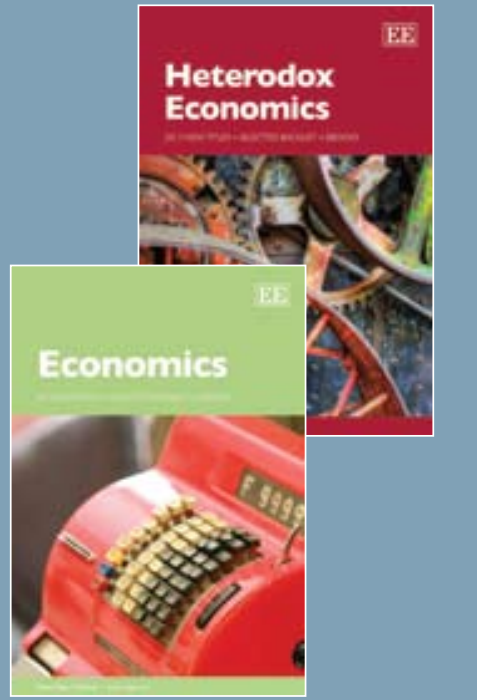

Google $=1$

ebooks ebrary eBooks dawsoners

eBooks com tor Ebook Library 